\title{
Rectification Performance of a Broadband RF-to-DC Rectifier on Wideband Pulses
}

\author{
H. S. Park, Student Member, IEEE, and S. K. Hong, Member, IEEE
}

\begin{abstract}
For wireless power transfer and energy harvesting applications, an advantage of having a broadband rectifier is the coverage of a wide range of narrowband frequencies as well as wideband pulses. Many broadband rectifiers have been recently proposed, but their reported characteristics are mainly evaluated at discrete frequencies. Due to the nonlinear nature of rectifiers, such frequency domain characteristics may not directly correspond to the time-domain, especially when input signals are wideband pulses. In this letter, we report the performance evaluation of a broadband rectifier on wideband pulses with various pulse parameters. Here, we use our recently proposed broadband rectifier as a test device. Using Gaussian-modulated RF pulses with various pulse parameters (i.e. pulsewidth, pulse repetition interval, and power), the rectification performance is verified via simulation and measurement. The results demonstrate sufficient rectification efficiency even for short pulses under appropriate parameters, where a maximum rectification efficiency of $71 \%$ is obtained.
\end{abstract}

Index Terms-Broadband, rectifier, efficiency, short pulse, wireless power transfer (WPT), energy harvesting, time-reversal

\section{INTRODUCTION}

$\mathrm{F}$ AR-FIELD wireless power transfer (WPT), which enables wireless powering of small, low-power devices in a range of applications (i.e. mobile, IoT, sensors, and biomedical), has been a topic of considerable interest in recent years [1]-[3]. Although many WPT and energy harvesting scenarios are based on using narrowband signals [4]-[6], WPT based on wideband pulses (i.e. time-reversed pulses, ultrawideband pulses) has also been proposed [7]-[11].

Since successful far-field WPT depends on efficient rectification as well as effective delivery RF power, RF-to-DC rectifier plays an important role in determining the efficiency of WPT [12]-[18]. In particular, a broadband rectifier may not only be utilized in rectifying narrowband signals in a wide frequency range, but also rectifying wideband short pulses.

While various broadband rectifiers with excellent bandwidth and rectification efficiency have been proposed [15]- [18], their reported performances were mainly evaluated at stepped discrete frequencies. However, due to the nonlinear nature of the rectifier, the rectification characteristics based on discrete frequencies is not linearly translated to time-domain wideband

Manuscript received --. This work was supported by National Research Foudation of Korea under grant 2017R1C1B5018179 and by Office of Naval Research Global under grant N62909-19-1-2049. (Correspondance: Sun K. Hong)

The authors are with School of Electronic Engineering, Soongsil University, Seoul 06978, Republic of Korea (e-mail: shong215@ssu.ac.kr).

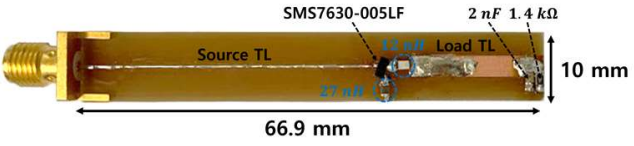

(a)

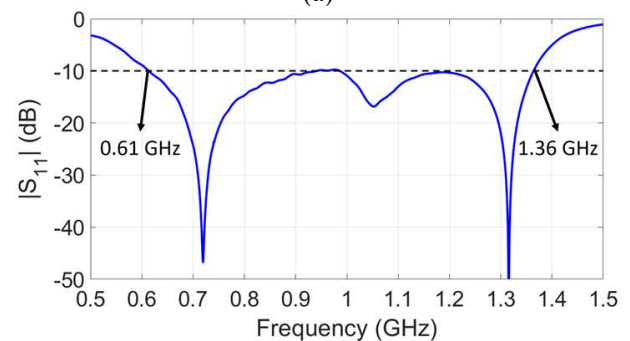

(b)

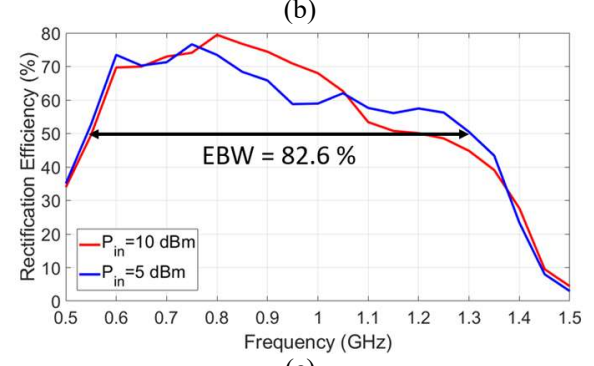

(c)

Fig. 1. Broadband RF-to-DC rectifier tested in this work [15]: (a) fabricated rectifier, (b) the reflection coefficient $\left(\left|S_{11}\right|\right)$ of the rectifier through stepped frequency sweep at $5 \mathrm{dBm}$ input power, and (c) the measured rectification efficiency through stepped frequency sweep.

pulses. In this regard, for WPT based on wideband pulses, an accurate performance evaluation of a broadband rectifier using actual wideband signals is essential.

In this letter, we demonstrate the rectification performance of our recently proposed broadband RF-to-DC rectifier [15] on short pulses with various pulse parameters (i.e. pulsewidth, pulse repetition interval, and power level) via simulation and measurement. In addition to demonstrating the rectification performance of the rectifier under test, it is hoped that the results from this letter will provide general insight into the behavior of a broadband rectifier with short pulses.

\section{BROADBAND RECTIFIER AND SHORT PULSE EXPERIMENT}

A recently developed broadband rectifier [15] is used as a test device in this work. This rectifier is based on a voltage doubler

Color versions of one or more of the figures in this communication are available online at http://ieeexplore.ieee.org.

Digital Object Identifier 10.1109/LMWC.2020.xxx 

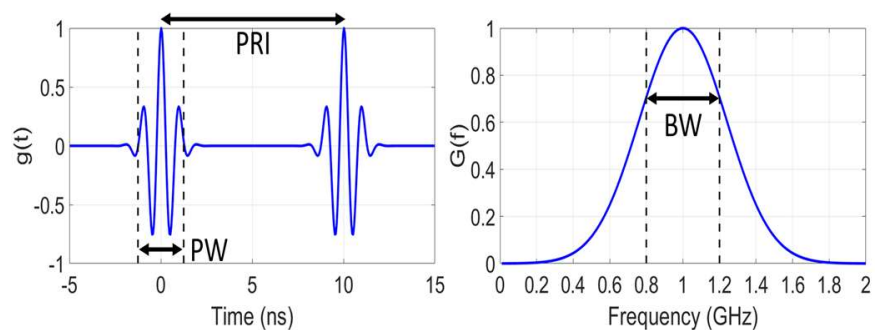

Fig. 2. An example Gaussian-modulated RF pulse (2.4 ns pulsewidth, $1 \mathrm{GHz}$ center frequency and $10 \mathrm{~ns}$ PRI): time-domain (left) and frequency-domain (right)

TABLE I. PULSE PARAMETERS TESTED

\begin{tabular}{|c|c|c|c|}
\hline $\begin{array}{c}\text { PW } \\
(\mathrm{ns})\end{array}$ & $\begin{array}{c}\text { BW } \\
(\mathrm{MHz})\end{array}$ & PRI (ns) & $\begin{array}{c}\text { Average } \\
\text { Input Power } \\
P_{\text {in,avg }}(\mathrm{dBm})\end{array}$ \\
\hline 10 & 100 & $20,25,30,35,40,45,50$ & \\
\hline 5 & 200 & $10,15,20,25,30,35,40,45,50$ & \multirow{2}{*}{$\begin{array}{c}\text {-20 to } 20 \\
\text { (increased } \\
\text { by 5) }\end{array}$} \\
\hline 3.33 & 300 & $10,15,20,25,30,35,40,45,50$ & \multirow{2}{*}{} \\
\hline 2.5 & 400 & $5,10,15,20,25,30,35,40,45,50$ & \\
\hline 2 & 500 & $5,10,15,20,25,30,35,40,45,50$ & \\
\hline 1.67 & 600 & $5,10,15,20,25,30,35,40,45,50$ & \\
\hline 1.43 & 700 & $3,5,10,15,20,25,30,35,40,45,50$ & \\
\hline
\end{tabular}

configuration, and the inductor connected to each SMS-7630 Schottky diode is the basis for broadband matching, as shown in Fig. 1(a). That is, an inductor in series with the diode cancels out the capacitance of the Schottky diode and generates multiple resonances, thereby enabling broadband matching to be achieved. Here, in the negative cycle of RF, charge is stored in the junction and package capacitance of the diode, so that the voltage doubler behavior is possible without the charging capacitor on the input side $[15,19]$.

Figs. 1(b) and 1(c) respectively show the reflection coefficient and rectification efficiency of the rectifier measured in a stepped frequency sweep. Here, an impedance bandwidth (IBW) of $76 \%(0.61-1.36 \mathrm{GHz})$ is obtained, which leads to a wide efficiency bandwidth (EBW) of $82.6 \%$ (where at least $50 \%$ of efficiency is maintained) at $5 \mathrm{dBm}$ input power. A more detailed design and frequency-domain performance of the broadband rectifier can be found in [15].

In order to evaluate the rectification performance on wideband pulses, we choose a train of Gaussian-modulated RF pulses as our signal type. Various pulse parameters, including pulsewidth (PW), pulse repetition interval (PRI), and RF average input power level $\left(P_{i n, a v g}\right)$, are used. The Fourier Transform $G(f)$ of a single Gaussian-modulated RF pulse $g(t)$ is expressed as

$$
G(f)=\frac{1}{\sigma \sqrt{2 \pi}} e^{-\frac{1}{2}\left(\frac{f-f_{0}}{\sigma}\right)^{2}},
$$

where $f_{0}$ is the center frequency and $\sigma$ determines the pulse bandwidth (BW) [20]. Since the rectifier under test has an IBW of $750 \mathrm{MHz}$ centered around $1 \mathrm{GHz}, f_{0}$ is set to $1 \mathrm{GHz}$ and $\mathrm{BW}$ is varied from $100 \mathrm{MHz}$ to $700 \mathrm{MHz}$ at a $100 \mathrm{MHz}$ increment to be completely included within the IBW of the rectifier.

Fig. 2 shows an example of normalized $g(t)$ and $G(f)$. Here, BW is a $-3 \mathrm{~dB}$ bandwidth and $\mathrm{PW}$ is defined to be the

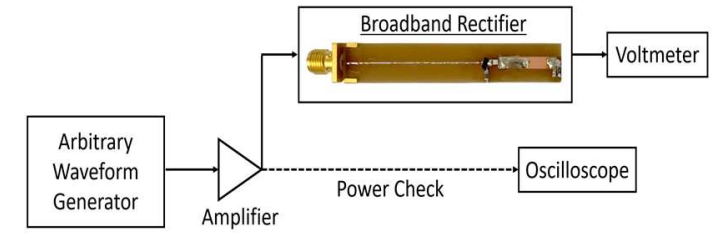

Fig. 3. Short pulse rectification measurement setup.

reciprocal of BW. To prevent the repeated pulses from overlapping, the minimum value of PRI is set to be at least twice the

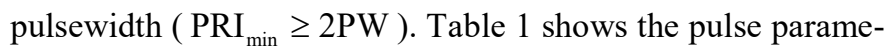
ters used in the experiment. The PRI values are set between the minimum value defined above and 50 ns. For each given PW (BW) and PRI pair, the pulse amplitude is varied so that a set of average power ranging from -20 to $20 \mathrm{dBm}$ are obtained. Setting the average power this way allows the same input power range as that used for discrete frequency measurements in [15], thereby providing a reasonable platform for comparison.

\section{RECTIFICATION RESULTS}

Using the abovementioned various input pulse parameters, rectification efficiency is obtained via simulation and measurement. The simulation is carried out with Advanced Design System. The experiment setup (Fig. 3) consists of an arbitrary waveform generator (Tektonix AWG7102) followed by an amplifier (Mini-circuits ZFL-2500VH+). Since the amplifier has a wide operating band $(10-2500 \mathrm{MHz})$, which ensures the bandwidth coverage all pulses tested. For each set of pulse parameters, the time domain signal is monitored with an oscilloscope (Tektonix TDS6154C) to ensure the proper signal amplitude for a given average power level. A voltmeter (Mastech MS8261) is used to measure the output DC voltage of the rectifier. The rectification efficiency $\eta$ can be defined as the ratio of the output DC power and average power of RF input, i.e.

$$
\eta=\frac{P_{D C}}{P_{i n, a v g}}=\frac{V_{D C}^{2}}{R_{L}} \frac{1}{P_{i n, a v g}},
$$

where $P_{D C}$ and $V_{D C}$, respectively, are the DC power and DC voltage delivered to the load and $R_{L}$ is the load resistance of $1.4 \mathrm{k} \Omega$ optimized for narrowband signals while providing sufficient RC time constant even for the longest PRI of $50 \mathrm{~ns}$.

Fig. 4 shows the simulated and measured $\eta$ versus the input average power and PRI for short pulses with different PWs. In order to make a visually effective comparison, different colors are used to represent the rectification efficiency at an increment of $10 \%$. In the measured plots, there is a small portion of blank spots (white color), which indicate unmeasured pulse parameters due to the output power limitation of the amplifier, which corresponds to a maximum peak voltage of about $5.6 \mathrm{~V}$. Therefore, the amplifier was limited to producing pulses with peak voltage less than this maximum value. In particular, at a given average input power, for shorter PW and longer PRI, the number of unmeasured pulses increases due to its higher peak voltage exceeding the maximum value. Nevertheless, the measured and simulated results in general show a good agreement exhibiting a similar trend. In both simulated and measured results, higher efficiency seems to occur with shorter PRIs. This can be 

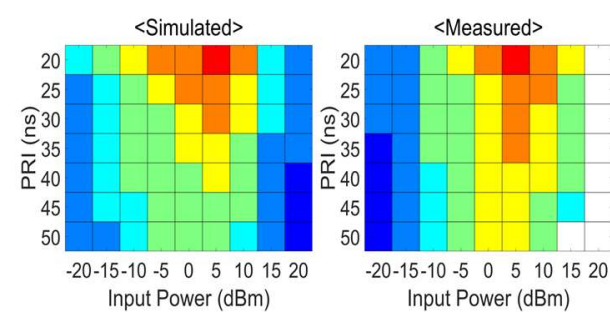

(a)
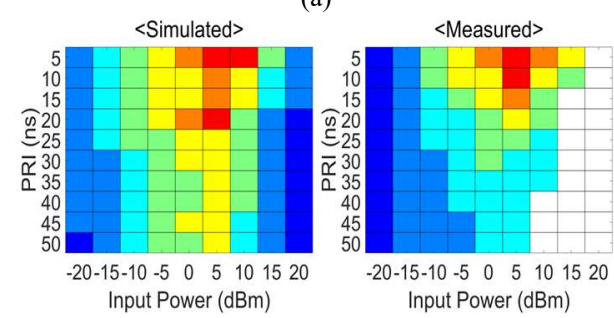

(d)
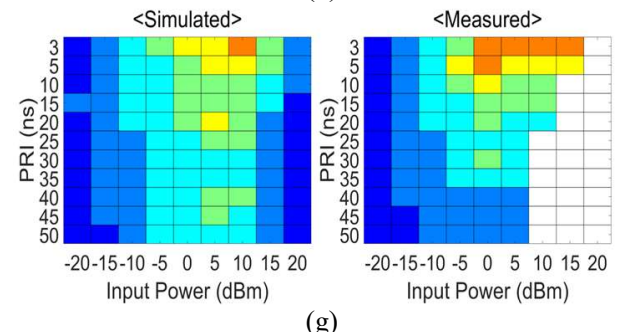
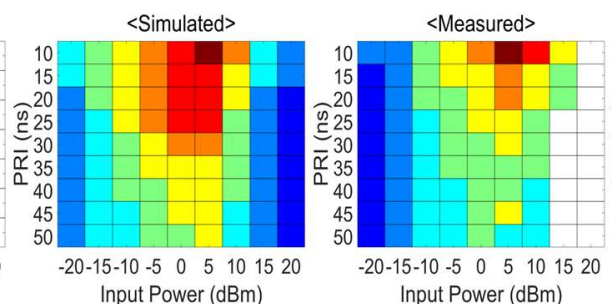

(b)
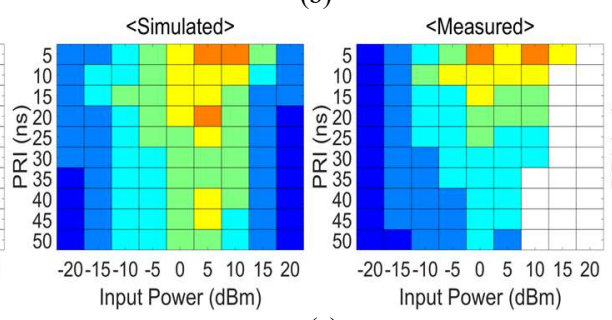

(e)
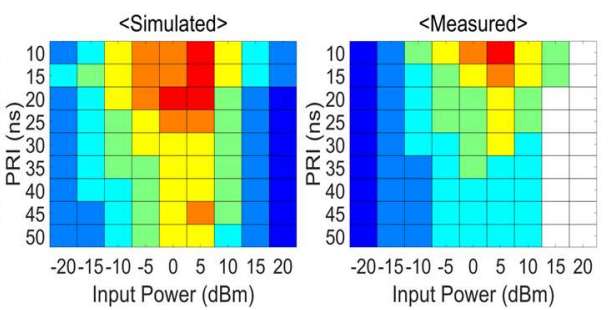

(c)
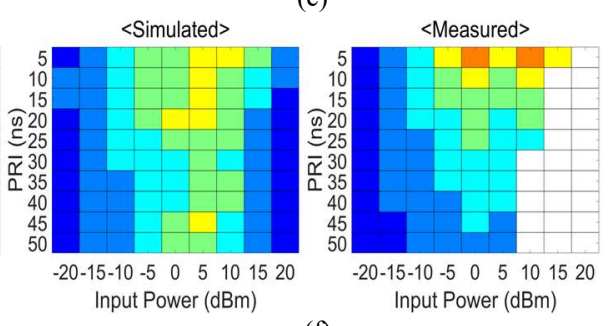

(f)

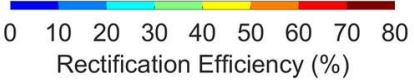

Fig. 4. Rectification efficiency versus PRI and input power for various PWs: (a) $10 \mathrm{~ns}$ (b) $5 \mathrm{~ns}$ (c) $3.33 \mathrm{~ns}$ (d) $2.5 \mathrm{~ns}$ (e) $2 \mathrm{~ns}$ (f) $1.67 \mathrm{~ns}$ (g) $1.43 \mathrm{~ns}$.

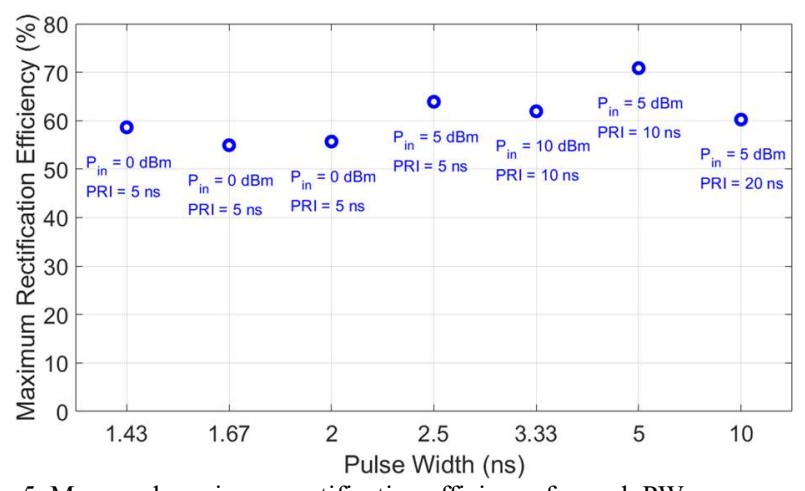

Fig. 5. Measured maximum rectification efficiency for each PW.

explained by the fact that the shorter the pulse interval, the sparser the power spectrum becomes in the frequency-domain. In other words, as PRI is shortened, the power spectrum has sparser peaks with higher amplitudes and the signal effectively becomes narrowband with less frequency content, which enables more stable rectification. At a given input average power, even though the peak voltage in the time-domain increases with longer PRIs to maintain the average power level, more frequency content (due to denser spectral peaks with lower amplitudes) causes rectification to be less efficient compared to the same pulse at lower PRIs. Moreover, a maximum efficiency seems to occur around an average input power ranging from 0 to $10 \mathrm{dBm}$ depending on PW. The reason for this variance (of the average input power level at which a maximum efficiency occurs) is that depending on PW, the time-domain pulses have different shapes, which causes them to reach the diode breakdown differently from each other.

The maximum measured rectification efficiency for each PW is plotted in Fig. 5. These results clearly show that for each PW, a maximum rectification efficiency occurs with the shortest PRI (except for the $1.43 \mathrm{~ns}$ pulse) and at different input average power levels. It can be seen that the values of maximum efficiency are generally maintained greater than $50 \%$, and a peak value of $71 \%$ occurs at $5 \mathrm{dBm}$ average power for $5 \mathrm{~ns} \mathrm{PW}$. In particular, for shorter PW, maximum efficiency of greater than $50 \%$ is achieved even at a relatively low input average power (at $0 \mathrm{dBm}$ ). Considering a maximum rectification efficiency of $76 \%$ at $5 \mathrm{dBm}$ input power for a n1.1 arrowband signal (see Fig. $1(c)$ ), the results in this study suggest that a rectification efficiency comparable to narrowband signals can be achieved for short pulses by selecting an appropriate PRI and power level. Furthermore, since the load resistance of the rectifier is originally optimized for narrowband signals, efficiency can be further improved by optimizing the load for short pulses.

\section{CONCLUSION}

The rectification performance of a broadband RF-to-DC rectifier on wideband pulses is tested and evaluated. The overall results demonstrate that the rectifier characteristics obtained at discrete frequencies do not linearly translate to short pulses due to the nonlinear nature of the rectifier as expected, highlighting the importance of an accurate investigation of the rectifier using actual short pulses to properly evaluate its rectification performance on short pulses. By using Gaussian-modulated RF pulses as input signals, it was shown that a rectification efficiency greater than $50 \%$ can be maintained with a maximum efficiency of $71 \%$. The results imply that sufficiently high rectification efficiency (comparable to that for narrowband input) can be achieved for short pulses under certain pulse conditions, validating the utility of a broadband rectifier for WPT based on wideband pulses. 


\section{REFERENCES}

[1] X. Lu, D. Niyato, P. Wang, and D. I. Kim, "Wireless charger networking for mobile devices: Fundamentals, standards, and applications," IEEE Wireless Commun., vol. 22, no. 2, pp. 126-135, Apr. 2015.

[2] H. Dinis, I. Colmiais, and P. Mendes, "Extending the limits of wireless power transfer to miniaturized implantable electronic devices," Micromachines, vol. 8, no. 12, p. 359, 2017.

[3] D. Belo, D. C. Ribeiro, P. Pinho, and N. B. Carvalho, "A selective, tracking, and power adaptive far-field wireless power transfer system," IEEE Trans. Microw. Theory Tech., vol. 67, no. 9, pp. 3856-3866, Sep. 2019.

[4] X. Wang, S. Sha, J. He, L. Guo, and M. Lu, "Wireless power delivery to low-power mobile devices based on retro-reflective beamforming," IEEE Antennas Wireless Propag. Lett., vol. 13, pp. 919-922, May 2014.

[5] J. He, X. Wang, L. Guo, S. Shen, and M. Lu, "A distributed retro-reflective beamformer for wireless power transmission," Microw. Opt. Technol. Lett., vol. 57, no. 8, pp. 1873-1876, Aug. 2015.

[6] P. S. Yedavalli, T. Riihonen, X. Wang, and J. M. Rabaey "Far-field RF wireless power transfer with blind adaptive beamforming for internet of things devices," IEEE Access, vol. 5, no. 7, pp. 1743-1752, Feb. 2017.

[7] S. K. Hong, V. M. Mendez, T. Koch, W. S. Wall, and S. M. Anlage, "Nonlinear electromagnetic time reversal in an open semireverberant system,'” Phys. Rev. A, Gen. Phys., vol. 2, no. 4, Oct. 2014, Art. no. 044013.

[8] R. Ibrahim, D. Voyer, A. Breard, J. Huillery, C. Vollaire, B. Allard, and Y. Zaatar, "Experiments of time-reversed pulse waves for wireless power transmission in an indoor environment," IEEE Trans. Microw. Theory Techn., vol. 64, no. 7, pp. 2159-2170, Jul. 2016.

[9] M.-L. Ku, Y. Han, H.-Q. Lai, Y. Chen, and K. J. R. Liu, "Power waveforming: Wireless power transfer beyond time reversal," IEEE Trans. Signal Process., vol. 64, no. 22, pp. 5819-5834, Nov. 2016.

[10] H. S. Park and S. K. Hong, "Investigation of time-reversal based farfield wireless power transfer from antenna array in a complex environment," IEEE Access, vol. 8, pp. 66517-66528, Apr. 2020.

[11] C.-L. Yang, Y.-L. Yang, and C.-C. Lo, "Subnanosecond pulse generators for impulsive wireless power transmission and reception," IEEE Trans. Circuits Syst. II, Exp. Briefs, vol. 58, no. 12, pp. 817-821, Dec. 2011

[12] E. Falkenstein, M. Roberg, and Z. Popovic, "'Low-power wireless power delivery,", IEEE Trans. Microw. Theory Techn., vol. 60, no. 7, pp. 2277-2286, Jul. 2012.

[13] A. Boaventura, D. Belo, R. Fernandes, A. Collado, A. Georgiadis, and N. B. Carvalho, "Boosting the efficiency: Unconventional waveform design for efficient wireless power transfer," IEEE Microw. Mag., vol. 16, no. 3, pp. 87-96, Apr. 2015.

[14] R. Ibrahim, D. Voyer, M. El Zoghbi, J. Huillery, A. Breard, C. Vollaire, B. Allard, and Y. Zaatar, "Novel design for a Rectenna to collect pulse waves at $2.4 \mathrm{GHz}$," IEEE Trans. Microw. Theory Techn., vol. 66, no. 1, pp. 357-365, Jan. 2018.

[15] H. S. Park and S. K. Hong, "Broadband RF-to-DC rectifier with uncomplicated matching network," IEEE Microw. Wireless Compon. Lett., vol. 30, no. 1, pp. 43-46, Jan. 2020.

[16] Y. L. Lin, X. Y. Zhang, Z.-X. Du, and Q. W. Lin, "Highefficiency microwave rectifier with extended operating bandwidth," IEEE Trans. Circuits Syst. II, Exp. Briefs, vol. 65, no. 7, pp. 819-823, Jul. 2018.

[17] P. Wu, S. Y. Huang, W. Zhou, and C. Liu, "One octave bandwidth rectifier with a frequency selective diode array," IEEE Microw. Wireless Compon. Lett., vol. 28, no. 11, pp. 1008-1010, Nov. 2018.

[18] M. M. Mansour and H. Kanaya, "High-efficient broadband CPW RF rectifier for wireless energy harvesting," IEEE Microw. Wireless Compon. Lett., vol. 29, no. 4, pp. 288-290, Apr. 2019.

[19] T. Mitani et al., "Analysis of voltage doubler behavior of $2.45-\mathrm{GHz}$ voltage doubler-type rectenna," IEEE Trans. Microw. Theory Tech., vol. 65, no. 4, pp. 1051-1057, Apr. 2017.

[20] R. L. Allen and D. Mills, Signal Analysis: Time, Frequency, Scale, and Structure. Hoboken, NJ, USA: Wiley, 2004. 